\title{
SMC X-1 As An Intermediate-Stage Flaring X-ray Pulsar
}

\author{
Dae-Sik Moon, Stephen S. Eikenberry, \& Ira M. Wasserman \\ Department of Astronomy \\ Space Science Building, Cornell University, Ithaca, NY 14853; \\ moon@astro.cornell.edu,eiken@astro.cornell.edu,ira@astro.cornell.edu
}

\begin{abstract}
We present Rossi X-ray Timing Explorer observations of the X-ray pulsar SMC X-1. The source is highly variable on short time scales $(<1 \mathrm{~h})$, exhibiting apparent X-ray flares occupying a significant fraction $(\sim 3 \%)$ of the total observing time, with a recurrence time of $\sim 100 \mathrm{~s}$. The flares seem to occur over all binary orbital phases, and correlate with the overall variability in the light curve. We find a total of 323 discrete flares which have a mean full width half maximum of $\sim 18$ s. The detailed properties of SMC X-1 do not vary significantly between the flares and the normal state, suggesting that the flare may be an extension of the normal state persistent emission with increased accretion rates. The flares resemble Type II X-ray bursts from GRO J1744-28. We discuss the origin of the SMC X-1 flares in terms of a viscous instability near the inner edge of the accretion disk around a weakly magnetized X-ray pulsar, and find this is consistent with the interpretation that SMC X-1 is in fact an intermediate-stage source like GRO J1744-28.
\end{abstract}

Subject headings: accretion,accretion disks — pulsars: individual (SMC X-1) — stars: neutron - X-rays: bursts - X-rays: stars

\section{Introduction}

Neutron star X-ray binaries are generally categorized into two groups: low mass X-ray binaries (LMXBs) and X-ray pulsars. The surface magnetic field of the central neutron star in an LMXB is thought to be $\sim 10^{8} \mathrm{G}$. The mass accretion with this magnetic field is most likely spherical, so that no significant inhomogeneity in the X-ray emission over the neutron star surface is expected - i.e., no persistent coherent pulsations are observed. The strong magnetic field, $\sim 10^{12} \mathrm{G}$, of an X-ray pulsar, on the other hand, can funnel the accretion matter onto the magnetic pole, which makes the central neutron star appear as a pulsar. 
Of particular interest are the so-called "intermediate-stage sources" speculated to lie between the LMXBs and X-ray pulsars, including "the Rapid Burster" (MXB 1730-355), GRO J1744-28 ("the Bursting Pulsar"), and SAX J1808.4-3658 ("the accreting millisecond pulsar") (Lewin et al. 1976; Fishman et al. 1995; in 't Zand et al. 1998). Both the Rapid Burster and GRO J1744-28 exhibit Type II X-ray bursts; however, only the former shows Type I X-ray bursts while only the latter has apparent coherent pulsations (Lewin et al. 1996). SAX J1808.4-3658, on the other hand, shows both Type I bursts and coherent pulsations (Wijnands \& van der Klis 1998; Chakarbarty \& Morgan 1998), but not Type II bursts. The magnetic field strengths of these sources have been inferred to be $\sim 10^{8-11} \mathrm{G}$, between those of LMXBs and X-ray pulsars.

Another possible intermediate-stage source is the X-ray pulsar SMC X-1, which has similar properties to GRO J1744-28, including its fast spin period ( $\sim 0.72$ s for SMC X-1; $\sim 0.47$ s for GRO J1744-28), steady spin-up, and inferred magnetic field $\left(\sim 10^{11} \mathrm{G}\right)$ (Bildsten \& Brown 1997; Li \& van den Heuvel 1997). In addition, once SMC X-1 was observed with an X-ray burst that resembles Type II bursts (Angelini, Stella, \& White 1991). It may be possible, therefore, that SMC X-1 and GRO J1744-28 belong to a distinctive group of X-ray binaries, "bursting pulsars", which show both coherent pulsations and Type II X-ray bursts (Li \& van den Heuvel 1997). To investigate this important possibility, we analyze all publicly available RXTE data for SMC X-1, searching for phenomena that may be related to X-ray bursts. We report that SMC X-1 in fact exhibits active flares resembling Type II bursts from GRO J1744-28.

\section{Data Analysis and a Flare Search}

We analyzed all publicly available RXTE Proportional Counter Array observations toward SMC X-1. Photon arrival times from the Good Xenon mode were transformed to the solar system barycenter using the JPL DE400 ephemeris. The Very Large Event Models were used to subtract backgrounds, and only the Standard 2 data obtained from the top xenon layers of Proportional Counter Units 0, 1, and 4 were considered for spectral analysis. Both the data within 30 minutes after passages through South Atlantic Anomaly and/or with a high $(>0.1)$ electron ratio were ignored.

The data toward SMC X-1 are occasionally contaminated by outbursts of the nearby ( 27' away) transient source XTE J0111.2-7317 (Chakrabarty et al. 1998). The contamination was easily identified by the source's $\sim 31$-s pulsations, with powers reach up to $>$ 5000 in Leahy-normalized PDSs. We searched for weak contamination in PDSs as narrow statistically significant ( $>10$ in Leahy-nomalized PDSs) peaks at $\sim 0.032 \mathrm{~Hz}$ (and/or at its 
harmonic frequencies). We excluded any data contaminated by XTE J0111.2-7317 from our analyses, as well as data containing dip-like features (e.g., Her X-1; Moon \& Eikenberry 2001 ) or close to the eclipse (i.e., binary orbital phase between 0.9 and 0.1 ) of SMC X-1. We obtained a total of 150 data segments with an average length of $\sim 1360$ s, giving a net observational duration of $\sim 204 \mathrm{ks}$. For spectral analysis, we considered only the spectrum between $2.5-25 \mathrm{keV}$ range due to a PCA responsivity problem (R. Remillard 2001, private communication), and assumed a systematic uncertainty of $1 \%$.

In our analysis, we define "flares" to be the part of a light curve that has three or more consecutive data bins with photon counts larger than a threshold value of $3 \sigma$ Poisson noise above the mean photon count in a given light curve. We analyzed all the 150 light curves as follows searching for the flares. First, we binned each light curve to 4-s time resolution and made a flare list. We extended the search with 2- and 8-s time resolutions, excluding the flares already found with different time resolutions. We found a total of 323 flares, and fit them a Gaussian function to estimate width and peak intensity. We obtained $\sim 18 \mathrm{~s}$ and $\sim 15$ s for the mean and rms standard deviation of FWHM; the reduced $\chi^{2}\left(=\chi_{\nu}^{2}\right)$ of the fits range from 0.5 to 1.9 . The total duration above half-maximum is $\sim 5.8 \mathrm{ks}$, indicating that SMC X-1 spends $\sim 3 \%$ of its time on flares.

\section{Flare Examples and Correlations with Other Parameters}

The rms variability of the 150 light curves ranges between 6 and $16 \%$, with a mean of $\sim 11 \%$. Figure 1 presents three light curves (and their PDSs) with very low (7\%), average (11 $\%)$, and very high (16\%) rms variability as examples representing three different variability levels. The mean photon count rates are $120.5 \pm 8.5,116.1 \pm 12.9$, and $146.4 \pm 23.3$, for Figure 1a, 1b, and 1c, respectively. No flare is found in Figure 1a, which has a low rms variability; three flares are found Figure 1b, which has an average rms variability. Strong flaring activity is well illustrated in Figure 1c, which has a high rms variability, with several apparent flares lasting for a few tens of seconds. At the flare peak, the photon count rate rises up to $\sim 2.5$ times of that outside the flares. The inset shows a Gaussian fit to the intense flare at $t \simeq 900 \mathrm{~s}$, with an FWHM estimated to be $\sim 23 \mathrm{~s}$. Leahy-normalized PDSs of the three light curves (Figure 1d, 1e, and 1f) show strong QPO-like peaks around 10 $\mathrm{mHz}$, as well as the peaks caused by the source's coherent pulsations at $\nu \simeq 1.4 \mathrm{~Hz}$ (and their harmonics), indicating the existence of $\sim 100$-s aperiodic variability independent of the flaring activity. The fractional $\mathrm{rms}$ (FRMS) amplitudes in the $2-50 \mathrm{mHz}$ range are $7 \pm 1,9$ \pm 1 , and $14 \pm 1 \%$ for $1 \mathrm{~d}, 1 \mathrm{e}$, and $1 \mathrm{f}$, respectively.

Figure 2 presents the pulse profiles of the three light curves, all showing the double- 
peaked, smooth profile typical of SMC X-1 (e.g., Levine et al. 1993). They have the same pulsed fractions of $\sim 39 \%$, with their second peaks at phase 0.56 with respect to the first ones at phase 0 . The ratio of the second peak to the first peak is $\sim 0.78$ for Figure 2a, while it is $\sim 0.96$ for $2 \mathrm{~b}$ and $2 \mathrm{c}$. No significant variation in the pulse peak ratio has been found within a given light curve, regardless whether it is obtained inside or outside flares.

Figure 3 compares the phase-averaged softness ratio, defined to be the ratio of the soft X-ray $(2-5 \mathrm{keV})$ photon count rates to those of the hard X-ray $(5-13 \mathrm{keV})$, of the three light curves in Figure 1 with the total photon count rates at $2-25 \mathrm{keV}$ with 4 -s resolution. The average softness ratio of the three light curves is invariant: $0.47 \pm 0.03,0.47 \pm 0.02$, and $0.46 \pm 0.04$ for Figure 1a, 1b, and 1c, respectively. This contrasts with the softness ratio of the LMC X-4 flares (Moon, Eikenberry, \& Wasserman 2002), which shows a strong linear correlation with the total photon count rate (inset in Figure 3).

We examined the spectral invariance of SMC X-1 over the flaring activity indicated by the constant softness ratio distribution more thoroughly via fitting the 32-s spectra obtained from the largest peak in each of the three light curves (i.e., the peak at $t \simeq 900 \mathrm{~s}$ in Figure $1 \mathrm{a}$, at $t \simeq 1050 \mathrm{~s}$ in $1 \mathrm{~b}$, and at $t \simeq 1110 \mathrm{~s}$ in $1 \mathrm{c}$ ) to a model spectrum. The model spectrum consists of a power-law component with a high-energy cutoff (for non-thermal magnetospheric emission) and a Gaussian component (for iron line emission), together with a component for photoelectric absorption by intervening interstellar matter. We fixed the central energy of the Gaussian component to be $6.7 \mathrm{keV}$ based on previous results (Angelini et al. 1991). Figure 4 compares the observed spectra with the best-fit model spectra, and Table 1 lists the best-fit parameters and $\chi_{\nu}^{2}$ of the fits. Although all parameters are poorly constrained, the power-law index does not change significantly over the flaring activity - consistent with the softness ratio distribution (Figure 3 ). The difference in $N_{\mathrm{H}}$ may be caused by the motion of the precessing, tilted accretion disk suggested to be responsible for the super-orbital motion of SMC X-1, but the low energy limit of the spectral fits (i.e., $2.5 \mathrm{keV}$ ) makes it difficult to constrain $N_{\mathrm{H}}$ propertly, because the photoelectric absorption by intervening interstellar matter is expected to be most significant in the soft energy band.

In order to perform statistical analyses, we calculated the flare fraction, which we define to be the ratio of the integrated time that SMC X-1 is flaring (i.e., within FWHMs of the Gaussian fits) to the total observing time of a given parameter, and investigate its correlation with the parameter. Figure 5 shows the distribution of the flare fraction as functions of the binary orbital phase (5a), rms variability of the light curve (5b), and the pulse peak ratio (5c). Some important results are worth noticing: the flare fraction (1) is larger than $1.4 \%$ over all the orbital phases (with its minimum at the orbital phase of $\sim 0.35$ ), (2) shows a significant variation from phase to phase, and (3) increases with the rms variability of the 
light curve, as well as with the pulse peak ratio. The correlation between flare fraction and rms variability identified in Figure 5b remains very similar when we use the flare-subtracted rms variability.

\section{Discussion}

SMC X-1 shows active flares that occupy $\sim 3 \%$ of the total observing time. The flares seem to occur over all binary orbital phases, and the flaring activity is proportional to the rms variability of the light curve (Figure 5). Except for the small change in the pulse peak ratio (Figure 2 and 5), no significant change is found along with the flaring activity. All these suggest that the SMC X-1 flares may be simple extensions of the persistent emission of a normal state with increased accretion rates, but without significant changes in the geometry of the accretion flows and the magnetosphere. Under the hypothesis that the double peaks in the pulse profile (Figure 2) are due to the two magnetic poles of SMC X-1, one simple explanation for the change in the pulse peak ratio may be that the increase in the accretion rate onto the fainter pole is higher than that onto the brighter pole during the flares. The invariant pulse peak ratio in a given light curve (regardless of flares), together with the correlation between the flare-subtracted rms variability and the flare fraction, on the other hand, may indicate the existence of variability whose time scale is longer than the flare recurrence time, $\sim 100 \mathrm{~s}$.

The SMC X-1 flares differ from Type I X-ray bursts from LMXBs for various reasons, including the shape of profiles and spectral properties. While the profile of Type I bursts shows an abrupt increase with an exponential decay in most cases, the SMC X-1 flares have symmetric Gaussian shapes. The X-ray spectrum of the SMC X-1 flare is far from thermal spectrum of Type I bursts (although the thermal spectrum of an X-ray pulsar is not well constrained, so it is not completely excluded that the SMC X-1 flare spectrum is thermal). In addition, the SMC X-1 spectrum does not show any apparent variation within a flare, while Type I burst shows spectral cooling as the burst continues. The changes in the softness ratio and pulse profile of the SMC X-1 flares differ from those of the LMC X-4 flares (Figure 3; Moon et al. 2002), which is one of the most well known and regular flaring X-ray binaries.

The SMC X-1 flares recall the Type II X-ray bursts found in GRO J1744-28 mainly due to the spectral invarince over the flaring activity. In fact, the burst spectrum of GRO J1744-28 was found to be very similar to that of SMC X-1, with a similar photon index of $\sim 1.2$ and e-folding energy of $\sim 14 \mathrm{keV}$ (Lewin et al. 1996). One difference is that the SMC X-1 flares lack the post-flare dip which often follows the Type II bursts from GRO J1744-28. The rather gradual rise of the SMC X-1 flares, however, may be responsible for it via offering 
sufficient time to replenish the material in the accretion disk. This is consistent with the interpretation that the SMC X-1 flares are simple extensions of a normal state with increased accretion rates, which is probably the most compelling argument for attributing the origin of the SMC X-1 flares to an accretion disk instability. Given the difference between GRO J1744-28 and SMC X-1 (i.e., a transient low-mass system versus a persistent high-mass one), we consider that the magnetic field strength, suggested to be comparable for the two sources, is critical to understanding the Type II bursts from GRO J1744-28 and the X-ray flares from SMC X-1.

We note that SMC X-1 may be capable of experiencing a viscous instability, namely the Lightman-Eardley instability (Lightman \& Eardley 1974), due to its $\sim 10^{29} \mathrm{G} \mathrm{cm}^{3}$ magnetic moment (Li \& van den Heuvel 1997). The radiation pressure in this case is comparable to the gas pressure around the inner disk radius (i.e., the magnetospheric radius), resulting in a viscous instability with slightly increased mass accretion (e.g., Cannizzo 1996, 1997). Because the instability develops near the inner edge of the accretion disk, it has an advantage in explaining bursts/flares with a short recurrence time. The viscosity parameter $(\alpha)$ of the classical $\alpha$-disk (Shakura \& Sunyaev 1973) at the transition radius between the "inner region"

and the "middle region" around an $1.4 M_{\odot}$ neutron star is $\alpha \simeq 216 t_{\text {vis }}^{-3 / 2} \dot{M}_{17}$, where $t_{\text {vis }}$ is the viscous time scale in seconds and $\dot{M}_{17}$ is the mass accretion rate in units of $10^{17} \mathrm{~g} \mathrm{~s}^{-1}$. For a typical value $\dot{M}_{17} \simeq 20$ for SMC X-1 (e.g., Wojdowski et al. 2000), the viscosity parameter is $\alpha \simeq 0.14$ and 4.4 when $t_{\text {vis }}$ is 1000 and $100 \mathrm{~s}$, respectively. The value 4.4 for $t_{\mathrm{vis}}=100 \mathrm{~s}$ is somewhat larger than generally expected, $\alpha \lesssim 1$. However, even larger values $(\alpha \simeq 10-100)$ were obtained for the dwarf nova HT Cas, possibly due to the patch nature of the accretion disk (Vrielmann, Hessman, \& Horne 2002). Alternately, the strong magnetic field of SMC $\mathrm{X}-1$ may help increase the viscosity parameter (R. Lovelace 2002, private communication). We need detailed numerical studies to investigate this scenario more thoroughly.

\section{Summary and Conclusions}

Through the analysis of the all publicly available RXTE data toward the X-ray pulsar SMC X-1, we find that the source is highly variable on short time scales $(<1 \mathrm{~h})$, and that Gaussian flares occur over all orbital phases with a $\sim 100$ s recurrence time scale. The flares occupy $\sim 3 \%$ of the total observing time, and the flaring activity is proportional to the overall variability of the source. While the pulse peak ratio shows a small systematic change along with the flaring activity, the PDSs, pulse profiles, softness ratios, and X-ray spectra during the flares are very similar to those outside the flares, indicating that the flares are probably extensions of a normal state just with increased accretion rates. This supports 
the interpretation that the SMC X-1 flares have their origin in an accretion disk instability and the suggestion that it may belong to a distinctive group of "bursting pulsars" with the "bursting pulsar" GRO J1744-28, owing to its $\sim 10^{11}$ G surface magnetic field. A viscous instability near the inner-edge of the accretion disk might be responsible for the SMC X-1 flares, although detailed studies on this scenario need to be done in the future.

We would like to thank the anonymous referee for the useful comments and suggestions. D.-S. M. acknowledges Akiko Shirakawa, Dong Lai, and Richard Lovelace for their comments. This research has made use of data obtained from the High Energy Astrophysics Science Archive Research Center provided by NASA's Goddard Space Flight Center. D.-S. M. is supported by NSF grant AST-9986898. S. S. E. is supported in part by an NSF Faculty Early Careeer Development award (NSF-9983830). I. M. W. is supported by the NASA grant NAG-5-8356.

\section{REFERENCES}

Angelini, L., Stella, L., \& White, N. E. 1991, ApJ, 371, 332

Bildsten, L., \& Brown, E. F. 1997, ApJ, 447, 897

Chakrabarty, D., \& Morgan, E. H. 1998, Nature, 394, 346

Chakrabarty, D., Levine, A. M., Clark, G. W., \& Takeshima, T. 1998, IAU Circ., No. 7048

Covino, S., Negueruela, I., Campana, S., Israel, G. L., Polcaro, V. F., Stella, L., \& Verrecchia, F. 2001, A\&A, 374, 1009

Fishman, G. J., et al. 1996, IAU Circ., No. 6290

in 't Zand, J. J. M., Heise, J., Muller, J. M., Bazzano, A., Cocchi, M., Natalucci, L., \& Ubertini, P. 1998, A\&A, 331, L25

Levine, A., Rappaport, S., Deeter, J. E., Boynton, P. E., \& Nagase, F. 1993, ApJ, 410,328

Lewin, W. H. G., et al. 1976, ApJ, 207, L95

Lewin, W. H. G., Rutledge, R. E., Kommers, J. M., van Paradijs, J., \& Kouvelitou, C. 1996, ApJ, 462, L39

Li, X. -D., \& van den Heuvel, E. P. J. 1997, A\&A, 321, L25 
Lightman, A. P., \& Eardley, D. M. 1974, ApJ, 187, L1

Moon, D. -S., \& Eikenberry, S. S. 2001, ApJ, 552, L135

Moon, D. -S., Eikenberry, S. S., \& Wasserman, I. M. 2002, ApJ, accepted

Rappaport, S., \& Joss, P. C. 1997, ApJ, 486, 435

Shakura, N. I., \& Sunyaev, R. A. 1973, A\&A, 24, 337

Vrielmann, S., Hessman, F. V., \& Horne, K. 2002, MNRAS, 332, 176

Wijnands, R., \& van der Klis, M. 1998, Nature, 394, 344

Wojdowski, P. S., Clark, G. W., \& Kallman, T. R. 2000, ApJ, 541, 963 
Table 1. Best-fit Parameters for Spectra Shown in Figure 4

\begin{tabular}{lrrr}
\hline \hline \multicolumn{1}{c}{ Parameter } & (a) & \multicolumn{1}{c}{ (b) } & \multicolumn{1}{c}{ (c) } \\
\hline$N_{\mathrm{H}}{ }^{*}\left(10^{22} \mathrm{~cm}^{-2}\right)$ & $3.2(0.9)$ & $1.6(1.5)$ & $2.2(0.9)$ \\
$\alpha^{*}$ & $1.6(0.3)$ & $1.3(0.5)$ & $1.6(0.3)$ \\
$E_{\mathrm{c}}(\mathrm{keV})^{*}$ & $17.2(7.1)$ & $6.9(3.2)$ & $14.3(5.2)$ \\
$E_{\mathrm{f}}(\mathrm{keV})^{*}$ & $7.8(6.9)$ & $12.6(10.2)$ & $14.0(10.6)$ \\
$\chi_{\nu}^{2 * *}$ & 0.81 & 1.3 & 0.81 \\
Flux $\left(10^{-9} \mathrm{ergs} \mathrm{cm}^{-2} \mathrm{~s}^{-1}\right)$ & 1.7 & 2.0 & 2.8 \\
\hline
\end{tabular}

${ }^{*} N_{\mathrm{H}}$ and $\alpha$ are the hydrogen nuclei column density of the intervening matter and the index of the power law component. $E_{\mathrm{c}}$ and $E_{\mathrm{f}}$ represent the cutoff and e-folding energy of the high-energy cutoff component.

**Each spectrum has 48 degrees of freedom.

Note. - Energy range is $2.5-25 \mathrm{keV}$, and the $90 \%$ uncertainty levels are quoted in the parentheses. 

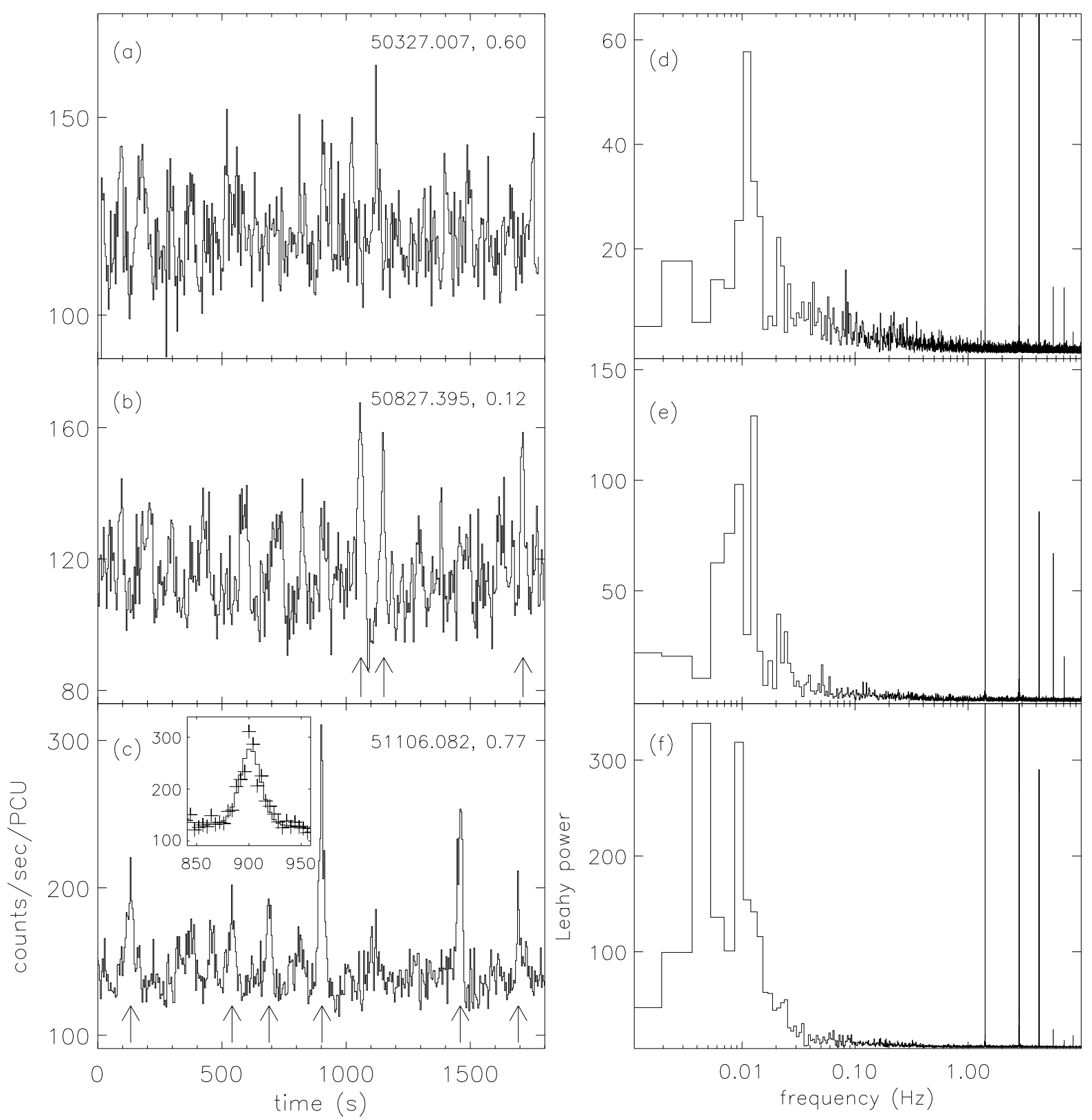

Fig. 1.- (a-c): Light curves (in the $2-25 \mathrm{keV}$ range) with 4 -s time resolution of three data segments that have $\sim 7 \%$ (a), $11 \%$ (b), and $\sim 16 \%$ (c) rms variabilities. The numbers in the upper-right corners represent the MJD of the beginning of each light curve and its binary orbital phase. The inset in (a) compares the intense flare (crosses) at $t \simeq 900 \mathrm{~s}$ with a Gaussian fit (solid histogram). The arrows indicate the central positions of nine flares. (d-f): Leahy-normalized PDSs of the three light curves in the left-hand side. 


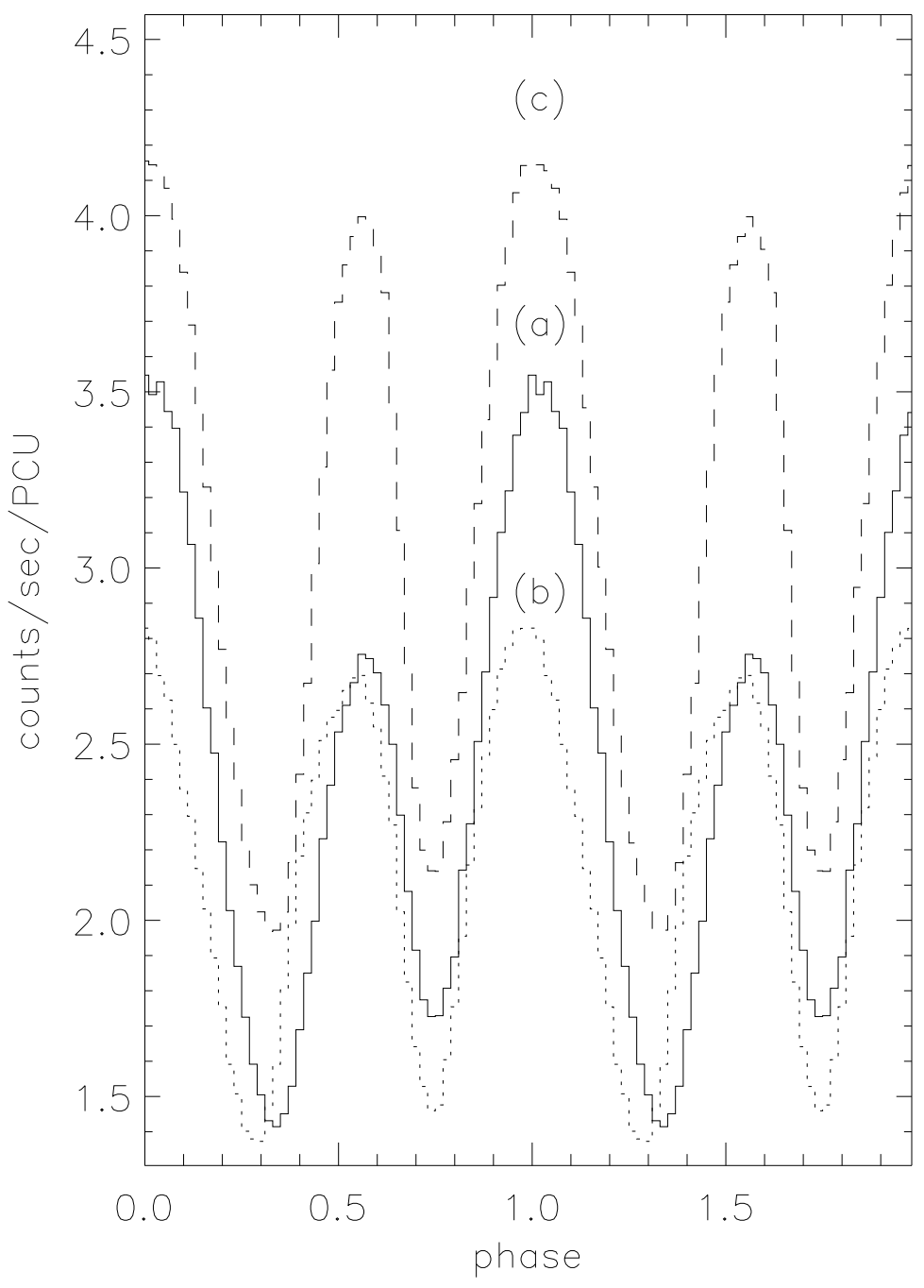

Fig. 2.- Pulse profiles of the three light curves in Figure 2 - solid line for 2a, dotted line for $2 \mathrm{~b}$, and dashed line for $2 \mathrm{c}$. The number of bin is 50 . 


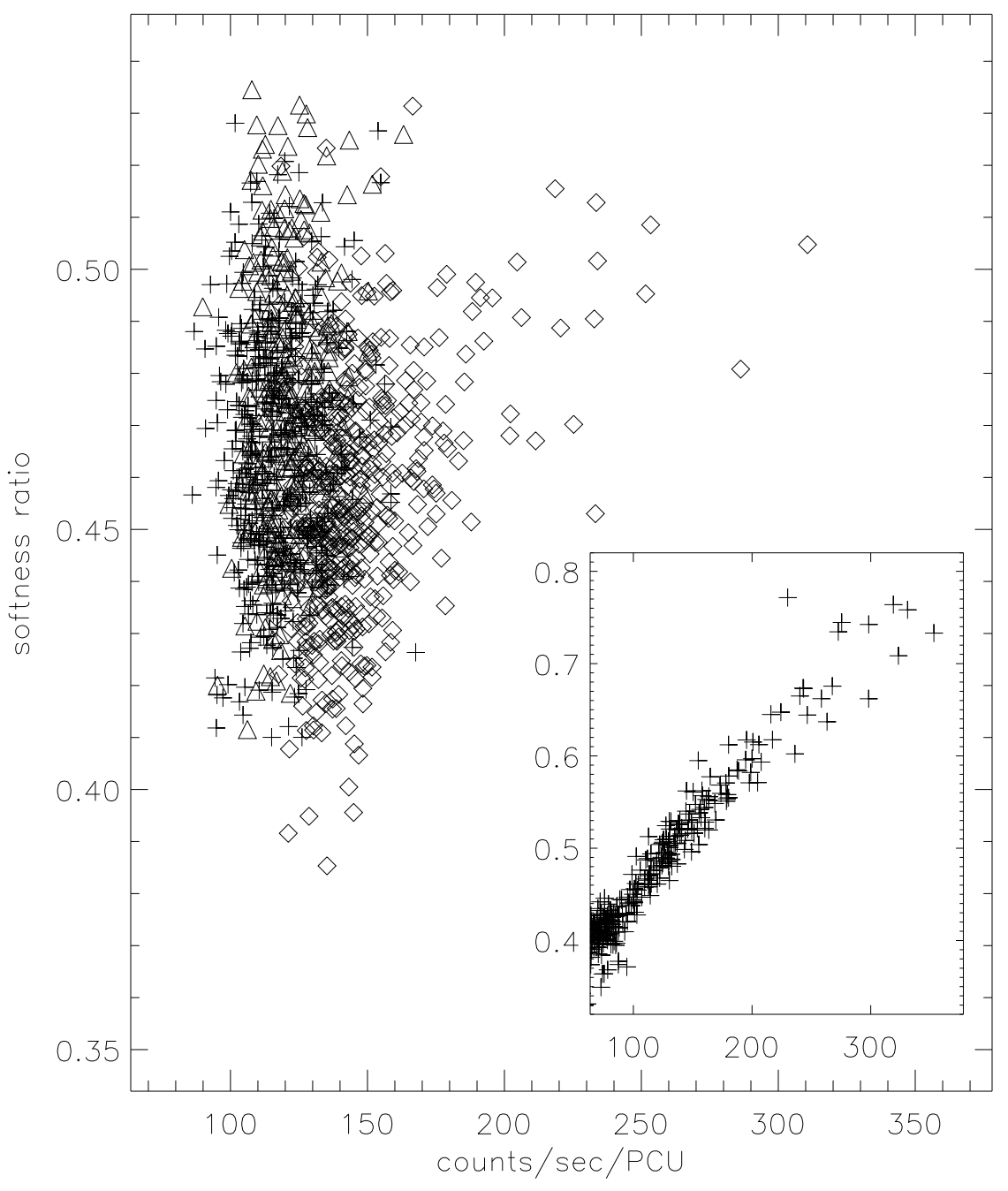

Fig. 3.- Softness ratio of the three light curves in Figure 1: triangle for 1a, crosses for 1b, and diamonds for 1c. The softness ratio is a ratio of the soft X-ray $(2-5 \mathrm{keV})$ photon count rates to those of hard X-ray $(5-13 \mathrm{keV})$. The inset shows the same softness ratio of the LMC X-4 flares (Moon et al. 2002). All the softness ratios are obtained with 4-s resolution. 

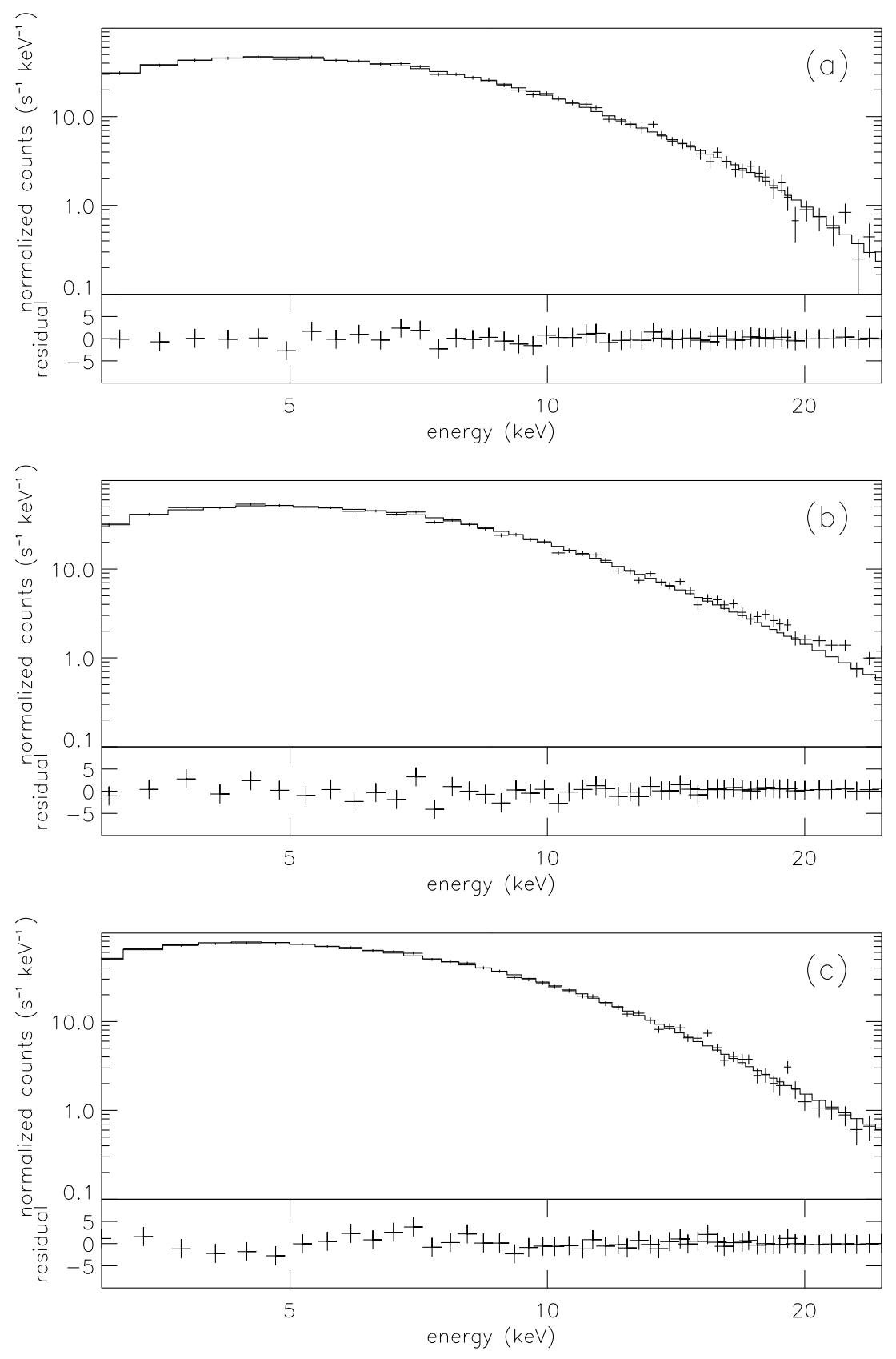

Fig. 4.- Comparison of the observed spectra (crosses) of the brightest peaks of the three light curves in Figure 1 (the peak at $t \simeq 900 \mathrm{~s}$ in $1 \mathrm{a}, t \simeq 1050 \mathrm{~s}$ in $1 \mathrm{~b}$, and $t \simeq 1110 \mathrm{~s}$ in $1 \mathrm{c}$ ) with the spectra (solid histogram) of the best-fit parameters. The bottom panels represent the residuals of the fittings. 

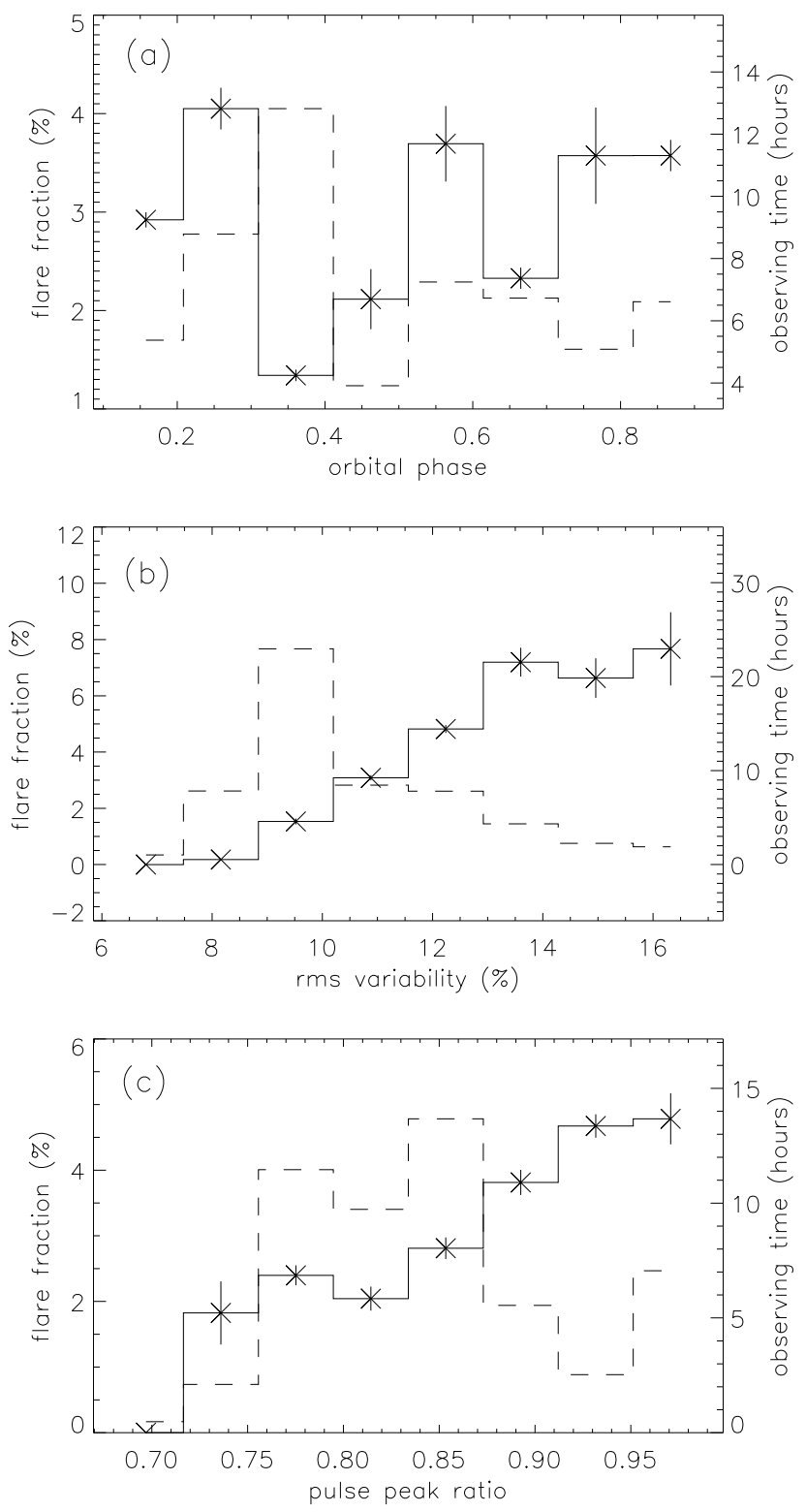

Fig. 5. - The distributions of the flare fraction (solid histograms) versus the binary orbital phase (a), the rms variability of the light curve (b), and the pulse peak ratio (c). The error bars correspond to 1- $\sigma$ confidence level. The dashed histograms represent the integrated observing time (the right-hand ordinates) in the corresponding binary orbital phase (a), the rms variability (b), and the pulse peak ratio (c). 